\title{
A RANDOMISED CONTROLLED STUDY OF COMPARISON OF INTRAOCULAR PRESSURE AND HAEMODYNAMIC RESPONSES TO INSERTION OF THE I-GEL, CLASSICAL LARYNGEAL MASK AIRWAY AND ENDOTRACHEAL TUBE
}

\author{
Aditya Khot ${ }^{1}$, P. B. Jamale2, V. K. Dhulkhed ${ }^{3}$, Shraddha Naik ${ }^{4}$ \\ ${ }^{1}$ Resident, Department of Anaesthesiology, Krishna Institute of Medical Sciences, Deemed University, Karad. \\ 2 Professor, Department of Anaesthesiology, Krishna Institute of Medical Sciences, Deemed University, Karad. \\ 3 Professor and HOD, Department of Anaesthesiology, Krishna Institute of Medical Sciences, Deemed University, Karad. \\ ${ }_{4}^{4}$ Assistant Professor, Department of Anaesthesiology, Krishna Institute of Medical Sciences, Deemed University, Karad.
}

\begin{tabular}{l}
\hline ABSTRACT \\
\hline BACKGROUND \\
The I-gel is a supraglottic airway device with soft gel like non-inflatable cuff. Compared to Classical LMA, I-GEL does not require \\
cuff inflation. I-gel works in harmony with the patient's anatomy, so that compression and displacement trauma are significantly \\
reduced. LMA and I-gel are supraglottic airways compared to Endotracheal Tube (ETT), which is inserted inside the trachea. This \\
study was done to determine the: 1. Intraocular pressure and 2. Haemodynamic response and compare it between I-gel insertion, \\
classical LMA insertion and laryngoscopy with endotracheal intubation.
\end{tabular}

\section{METHODS}

120 adult patients were allocated to either I-GEL, C-LMA or ETT group with 40 patients in each group. All three devices were introduced with standard technique. The outcomes measured were increased in IOP (Intraocular pressure), haemodynamic parameters namely Systolic Blood pressure (SBP), Diastolic Blood Pressure (DBP), Heart Rate (HR) after insertion.

\section{RESULTS}

Insertion of the I-gel did not increase Intraocular Pressure. Insertion of an endotracheal tube increased IOP from $11.53 \pm 1.3$ to $18.36 \pm 1.6 \mathrm{mmHg}(\mathrm{P}<0.001)$. The post-insertion IOP exceeded the pre-induction value $12.5+1.4$. Insertion of the LMA increased IOP from $11.65 \pm 1.29$ to $13.5 \pm 1.88 \mathrm{mmHg}(\mathrm{P}<0.001)$, did exceed the pre-induction value $(12.57+1.39)$ but slightly. Tracheal intubation significantly increased HR, SBP and DBP. Insertion of the LMA significantly increased HR and SBP. These increases were significantly higher than those, which followed insertion of the I-gel device.

\section{CONCLUSION}

Insertion of I-gel device provides better stability of IOP and haemodynamic system compared to ETT or LMA undergoing elective non-ophthalmic surgery.

\section{KEYWORDS}

I-GEL, Classical LMA, Intraocular Pressure, Endotracheal tube, Haemodynamic Response.

HOW TO CITE THIS ARTICLE: Khot A, Jamale PB, Dhulkhed VK, et al. A randomised controlled study of comparison of intraocular pressure and haemodynamic responses to insertion of the I-gel, classical laryngeal mask airway and endotracheal tube. J. Evolution Med. Dent. Sci. 2016;5(41):2514-2519, D0I: 10.14260/jemds/2016/587

\section{INTRODUCTION}

Laryngoscopy and endotracheal intubation is the commonest method of securing a definitive airway for administering anaesthesia. However, it is associated with tachycardia and hypertension. ${ }^{1}$ and an increase in intraocular pressure. 2,3 These changes have been observed to be associated with increased catecholamine levels confirming a predominantly sympathetic response to it. The rise in IOP may be secondary to increased sympathetic activity causing vasoconstriction and an increase in central venous pressure, which has a closer relationship with intraocular pressure than systemic arterial pressure. ${ }^{4}$

Financial or Other, Competing Interest: None.

Submission 07-04-2016, Peer Review 29-04-2016,

Acceptance 07-05-2016, Published 21-05-2016.

Corresponding Author:

Dr. Aditya Khot,

Koyna Vasahat

Karad-415110,

Satara District,

Maharashtra.

E-mail: adityakhot87@gmail.com

DOI: $10.14260 /$ jemds/2016/587
Transitory hypertension and tachycardia are of no consequence in healthy individuals, but they may be hazardous to the patients with hypertension, myocardial insufficiency or cerebrovascular disease.

The rise in intraocular pressure during laryngoscopy and intubation is a matter of concern in patients of acute glaucoma and open eye injuries where even small increase in intraocular pressure sustained for a short time may change what is critical disc perfusion into disc ischaemia and extrusion of the contents of the eye ball with possible resultant blindness. ${ }^{4}$

Supraglottic airway devices are now widely used for surgery requiring general anaesthesia, so as to avoid the complications associated with tracheal intubation. ${ }^{5}$ Dr. Archie Brain, a British anaesthesiologist, for the first time introduced the laryngeal mask airway in 1983, which is a supraglottic airway device with an inflatable cuff forming a low pressure seal around the laryngeal inlet and permitting ventilation. ${ }^{6}$ Several refinements of Brain's original prototype have led to development of newer supraglottic airway. I-Gel is the single use supraglottic airway introduced by Dr. Muhammed Aslam Nasir in 2007 and manufactured by intersurgical, UK (Intersurgical Ltd., Wokingham, Berkshire, UK). ${ }^{7}$ 


\section{AIMS AND OBJECTIVES}

To Determine the,

1. Change in Intraocular pressure,

2. Change in haemodynamic response and compare it between I-Gel insertion, classical laryngeal mask airway insertion and laryngoscopy with endotracheal intubation.

\section{MATERIAL AND METHODS}

\section{Source of Data (Sample)}

The study was conducted at Krishna Hospital, Karad.

\section{Design of the Study}

Randomised clinical trial.

\section{Inclusion Criteria}

Surgical candidates both male and female aged 15-60 years, ASA grade I or II, Mallampati grade I or II, presenting for elective non-ophthalmic surgery requiring general anaesthesia.

\section{Exclusion Criteria}

- Presence of any significant acute or chronic lung disease.

- Pathology of the neck or upper respiratory tract.

- Potential difficult intubation.

- Increased risk of aspiration (Hiatus hernia, gastrooesophageal reflux or full stomach).

- Pregnant women.

- $\quad$ BMI $>30$.

- Ocular hypertension patients.

- Glaucoma patients.

A total of 120 patients were included in the study. Three groups were formed of 40 cases each. Group 1 - I-GEL $(n=40)$, Group 2 - C-LMA (n=40), Group 3 - ETT $(n=40)$.

Following Departmental Research Committee and Institutional Ethical Board approval, written informed consent were obtained.

\section{Technique}

The patient was shifted to operation theatre, all standard monitors were attached and all baseline parameters were noted including the intraocular pressure (With the use of Schiotz tonometer after instilling two drops of $4 \%$ lidocaine in each eye). Following pre-oxygenation for 3 minutes, anaesthesia was induced with Injection Thiopentone $5 \mathrm{mg} / \mathrm{kg}$. Check ventilation was performed before giving an intubating dose of Inj. Vecuronium $0.1 \mathrm{mg} / \mathrm{kg}$. Patients were ventilated using a face mask for three minutes before insertion of any of the devices. The level of adequate anaesthesia was assessed by loss of verbal contact with the patient, loss of eyelash reflex and relaxation of jaw. The size of the device was decided by anaesthetist based on patient's body weight and manufacturer's recommendation, and all the standard pre use tests were performed. Once adequate depth was achieved all the parameter readings were noted down and then each device was inserted. Following insertion of each device, successful placement was checked by chest expansion, reservoir bag movement and appearance of capnographic tracing.

Surgical incision was requested after five minutes of airway manipulation to avoid likely stimuli.

\section{Parameters Studied During the Procedure}

1. Intraocular Pressure in $\mathrm{mmHg}$.

2. Haemodynamic Parameters.

The following Haemodynamic Parameters Were Recorded in all Patients

- Heart Rate [HR] in beats per minute.

- $\quad$ Systolic Blood Pressure [SBP] in mmHg.

- Diastolic Blood Pressure [DBP] in mmHg.

\section{These were Recorded}

- Before induction.

- Before insertion of device.

- Just after insertion of device.

- 2 min after insertion of device.

- 5 min after insertion of device.

\section{OBSERVATION AND RESULTS}

Age, gender and body weight distribution were comparable between all the groups. Statistical tests used were repeated measures ANOVA test with statistical software GraphPad InStat 3.06 and Tukey Kramer multiple comparisons tests for individual group comparisons (This test was applied only when significant difference was appreciated by ANOVA test).

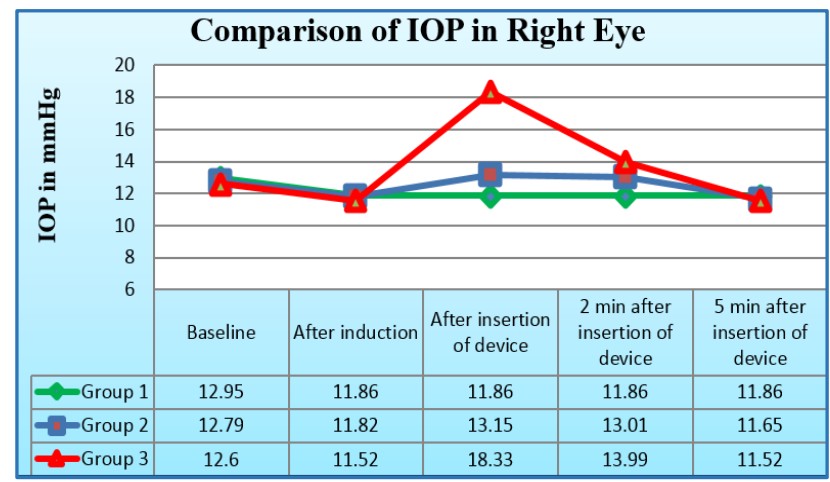

Fig. 1

The right eye intraocular pressure showed significant rise with ETT group as compared to LMA and I-GEL group at the time of insertion of device and $2 \mathrm{~min}$ after insertion. (P value $<0.0001$, extremely significant).

The Tukey Kramer multiple comparisons test showed that there was significant difference in the mean right eye IOP between all comparisons suggesting that the IOP levels after insertion of C-LMA and ETT remained raised even after 2 mins of insertion as compared to those after insertion of I-GEL.

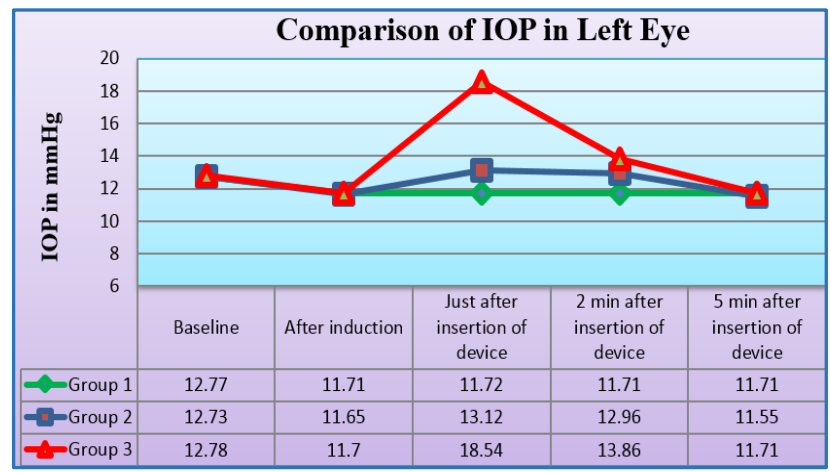

Fig. 2 
The left eye intraocular pressure showed significant rise with ETT group as compared to LMA and I-GEL group at the time of insertion of device and 2 mins after insertion ( $P$ value $<0.0001$, extremely significant).

The Tukey Kramer multiple comparisons test showed that there was significant difference in the mean left eye IOP between all comparisons suggesting that the IOP levels after insertion of C-LMA and ETT remained raised even after 2 mins of insertion as compared to those after insertion of I-GEL.

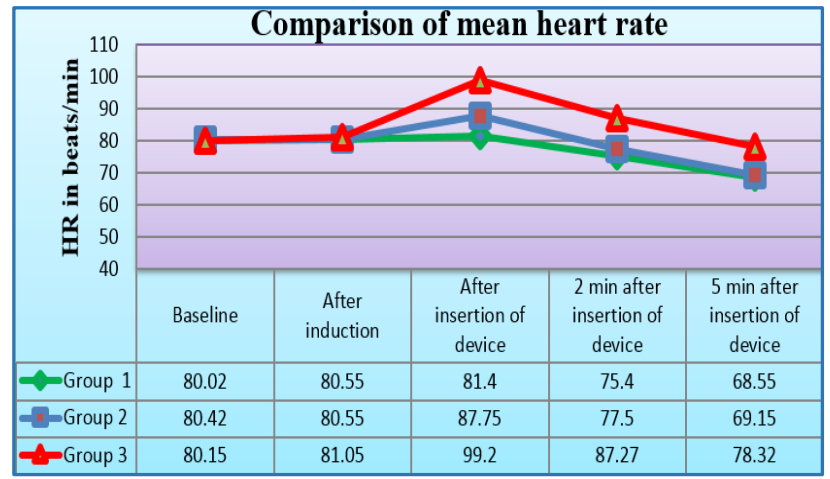

Fig. 3

The mean heart rate showed significant rise with ETT group as compared to LMA and I-GEL group at the time of insertion of device, $2 \mathrm{~min}$ after insertion and $5 \mathrm{~min}$ after insertion. ( $\mathrm{P}$ value $<0.0001$, extremely significant).

The Tukey Kramer multiple comparisons test showed significant differences in all comparisons at the time of insertion, 2 mins after insertion and 5 mins after insertion, except that it showed significant difference between C-LMA and I-GEL insertion at 2 mins and 5 mins after insertion. This suggests that the HR levels remained raised even 2 mins and 5 mins after insertion of ETT as compared to the HR levels after insertion of C-LMA and I-GEL.

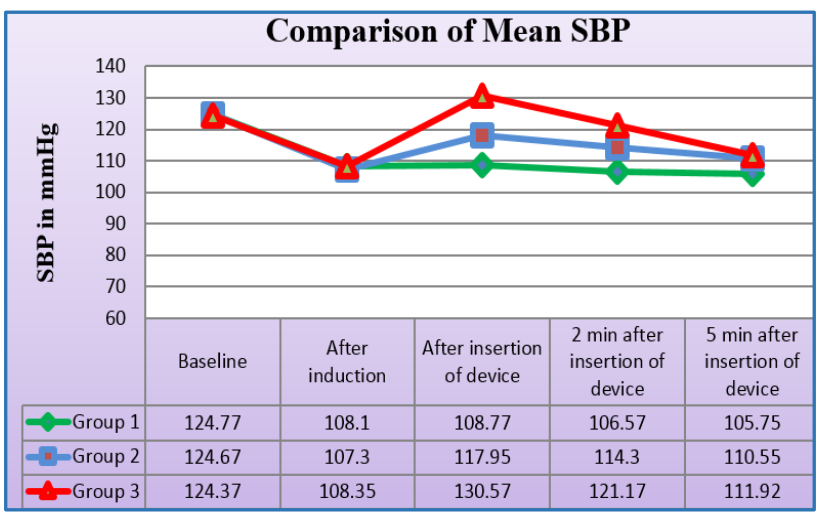

Fig. 4

The mean systolic blood pressure showed significant rise with ETT group as compared to LMA and I-GEL group at the time of insertion of device, 2 mins after insertion and 5 mins after insertion. (P value $<0.0001$ ).

The Tukey Kramer multiple comparisons test showed significant differences in all comparisons at the time of insertion and 2 mins after insertion and 5 mins after insertion, except that it showed no significant difference between ETT and C-LMA insertion 5 mins after insertion. This suggests that the SBP levels remained raised even 5 mins after insertion of
ETT as compared to the SBP levels after insertion of C-LMA and I-GEL.

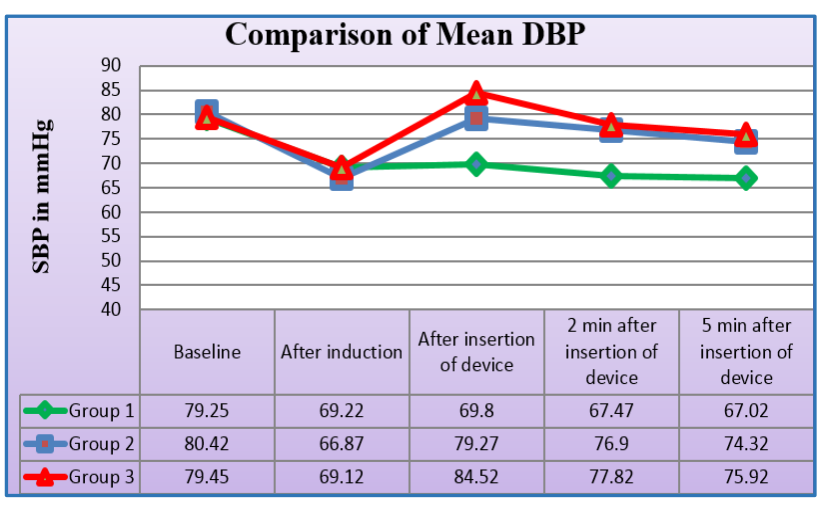

Fig. 5

The mean diastolic blood pressure showed significant rise with ETT group as compared to LMA and I-GEL group at the time of insertion of device, 2 mins after insertion and 5 mins after insertion. (P value $<0.0001$ ).

The Tukey Kramer multiple comparisons test showed significant differences in all comparisons at the time of insertion, 2 mins after insertion and 5 mins after insertion, except that it showed no significant difference between ETT and C-LMA insertion at the time of insertion, 2 mins and 5 mins after insertion. This suggests that the DBP levels remained raised consistently from insertion till even 5 mins after insertion of ETT as well as C-LMA as compared to the DBP levels after insertion of I-GEL.

\section{DISCUSSION}

Present study reveals that the increase in intraocular pressure, heart rate and blood pressure after insertion of an airway device compared to the baseline values was least after I-GEL use in comparison with classical LMA and conventional tracheal tube intubation.

Laryngoscopy and tracheal intubation to achieve airway control in anaesthesia practice have been consistently bothering anaesthesiologists with regard to regular occurrence of the pressor responses associated with it. The haemodynamic responses manifesting as increase in heart rate and blood pressure are due to reflex sympathoadrenal discharge provoked by epilaryngeal and laryngotracheal stimulation subsequent to laryngoscopy and tracheal intubation. ${ }^{8}$ the stress response to tracheal intubation and extubation is also associated with increase in IOP. ${ }^{9}$

Among the factors affecting the intraocular pressure, changes in the systemic arterial blood pressure have the lowest significance owing to displacement of aqueous from the anterior chamber, which is equilibrated by a shift of blood from the choroidal vessels.10,11 A direct and immediate relation exists between the central venous pressure and intraocular pressure and the rise in central venous pressure caused by coughing is instantly transmitted to the eye by impeding the efflux of aqueous. ${ }^{10,11}$

Laryngoscopy and intubation as shown in this study also have an important role in raising intraocular pressure, possibly as a result of the sympathetically mediated vasoconstriction generating increased venous return and a sudden rise in central venous pressure. ${ }^{12}$ 
The acute increase in IOP may be dangerous for patients with impending perforation of eye, perforating eye injuries, glaucoma, etc.

This problem has drawn the attention of many workers to study the attenuation of these responses with some pretreatment or by some alternative to laryngoscopy and tracheal intubation, viz. LMA. Lignocaine pre-treatment, either intravenous or nebulised, has been used to attenuate ocular and systemic responses to laryngoscopy and tracheal intubation. ${ }^{33,14,15}$

LMA, as an alternative to endotracheal tube has attracted the attention of many workers with regards to IOP changes, as it obviates the need for laryngoscopy and tracheal intubation. Holden et $\mathrm{al}^{16}$ were the first one to compare the IOP changes using LMA and endotracheal tube and their observations as well as those of Lamb et al ${ }^{17}$ revealed a significantly smaller increase in IOP using LMA both on placement and removal as compared to endotracheal intubation. Similar results were reported by Whitford et al ${ }^{18}$ and Duman et al. ${ }^{19}$

The I-GEL is a new supraglottic device without an inflatable cuff, designed for use during anaesthesia. ${ }^{20}$ It is a latex free, disposable device, made of a medical grade thermoplastic elastomer. I-GEL is anatomically preformed to mirror the perilaryngeal structures. The device contains an epiglottis blocker, which helps to prevent epiglottis from downfolding or obstructing laryngeal inlet. The soft noninflatable cuff seals anatomically against perilaryngeal structures. $^{21}$

In our study the baseline IOP, HR, SBP and DBP values were comparable among all the groups with no significant difference between them.

The rise in IOP after insertion of device was greater for the tracheal tube intubation group $(18.33+1.92$ in right eye and $18.54+2.0$ in left eye) than for the LMA group $(13.15+1.85$ in right eye and 13.12+1.61 in left eye) and I-GEL group where there was no rise at all $(11.86+1.61$ in right eye and $11.72+1.60$ in left eye) [p value $<0.001$.

The rise in HR after insertion of device was highest in ETT group $(99.2 \pm 8.39)$ followed by C-LMA group $(87.75 \pm 10.35)$ and almost no rise in I-GEL group $(81.4+11.81)$ [p value $<0.001]$.

The rise in SBP after insertion of device compared to after induction values $(108.1+9.71,107.17+9.14,108.05+7.23$ for IGEL, C-LMA and ETT respectively) was highest in ETT group (130.57 \pm 8.16$)$ followed by classical LMA group (117.95 \pm 7.39 ) (which did not rise above pre-induction value) and almost no rise in I-GEL group $(108.77+9.10)$ [p value $<0.001$ ].

The rise in DBP after insertion of device compared to after induction values $(69.22+9.82,66.87+9.65,69.12+9.72$ for IGEL, C-LMA and ETT, respectively) was highest in ETT group (84.52 \pm 11.78 ) followed by LMA group (79.27 \pm 7.31 ) (which did not rise above pre-induction value) and almost no rise in IGEL group $(69.8+10.01)$ [p value $<0.001$ ]

In a similar study by Ismail et al, ${ }^{22}$ they found that insertion of the I-GEL did not increase IOP. Insertion of an endotracheal tube increased IOP from $11.6 \pm 1.6$ to $16.5 \pm 1.7 \mathrm{mmHg}$ $(\mathrm{p}<0.001)$. The post-insertion IOP exceeded the pre-induction value $(\mathrm{p}<0.05)$. Insertion of the LMA increased IOP from $13.0 \pm 1.5$ to $14.7 \pm 1.8 \mathrm{mmHg}(\mathrm{p}<0.01)$, but this did not exceed the pre-induction value. Tracheal intubation significantly increased HR, SBP and DBP. Insertion of the LMA significantly increased HR and SBP. These increases were significantly higher than those which followed insertion of the I-GEL device.

In another study conducted by Garima Agarwal et al, ${ }^{23}$ they compared rise in heart rate, mean arterial pressure and IOP in paediatric patients after use of Proseal LMA (P-LMA) and tracheal tube. They reported that there was significantly high rise in heart rate and mean arterial pressure from baseline in paediatric patients with use of LMA and tracheal tube. There was significant increase in heart rate and blood pressure in tracheal tube group compared to P-LMA group of patients. In their study there was no significant difference in the heart rate $(\mathrm{P}=0.476)$, mean blood pressure $(\mathrm{P}=0.578)$ and IOP $(\mathrm{P}=0.998)$ before insertion of the airway device between the two groups. Following insertion of endotracheal tube, there was a highly significant rise in heart rate $(\mathrm{P}<0.001)$, mean blood pressure $(\mathrm{P}<0.001)$ and IOP $(\mathrm{P}<0.001)$. Though there was no significant rise in the heart rate $(\mathrm{P}=0.921)$ and mean blood pressure $(\mathrm{P}=0.327)$, there was a significant rise in IOP $(\mathrm{P}=0.007)$ in group P-LMA after insertion. The percentage change in heart rate and mean blood pressure when compared between the two groups was found to be significant and highly significant, respectively.

In our study also endotracheal tube insertion showed significant rise in heart rate and blood pressure and intraocular pressure, which was less in LMA group. In addition to this we also studied the response to I-GEL insertion, which showed no rise at all in IOP, HR, SBP and DBP.

In another study conducted by Maharajan SK.24, they have compared haemodynamic variables of I-GEL and laryngeal mask airway with tracheal intubation during laparoscopic surgery. In their study there was increase in mean heart rate after use of airway device in all three groups, but maximum with tracheal tube and least with I-GEL. While basal heart rate and heart rate prior to airway placement were comparable among all three groups, there was significant difference among the groups after one minute of use of airway device ( $\mathrm{p}$ value $<0.001$ ). Heart rate increment was also there after three and five minutes of airway manipulation, but the difference among three groups was statistically not significant. Systolic Blood Pressure (SBP) increment was also maximum with tracheal intubation, moderate with LMA and least with I-GEL. Here again starting with similar baseline and prior to placement readings, there was highly significant difference in SBP among the groups after one minute of airway placement ( $\mathrm{p}=0.002)$. Comparisons of systolic blood pressure in other time intervals in three groups were statistically not significant. Regarding diastolic blood pressure, there was significant difference in one minute after insertion ( $\mathrm{p}=0.019)$, but no significant difference detected in other time intervals studied.

Our study also showed similar results except that the rise in HR, SBP, DBP even after 5 mins were significantly higher statistically in ETT and LMA group (Although decreased from just after insertion values) when compared to I-GEL group. This might be because of the use of Inj. propofol as the induction agent as compared to Inj. thiopentone used by us. Also we have compared intraocular pressure as one of the parameters.

Jindal $\mathrm{P}$ et al ${ }^{25}$ compared haemodynamic effects of three supraglottic airway devices I-GEL, LMA and Streamlined Pharyngeal Airway (SLIPA), which is also a supraglottic airway without inflatable cuff during general anaesthesia with muscle relaxation. In their study, they found that there was no 
significant change in heart rate in group I-GEL and in group SLIPA at any time.

On comparing group I-GEL to group LMA, there were significant changes in HR post insertion till 5 mins. In all three groups, there was significant difference in systolic blood pressure and diastolic blood pressure from insertion of device till 5 mins after insertion.

In our study we have used endotracheal tube which shows the highest rise in HR, SBP and DBP. Comparison between LMA and I-GEL showed results similar to this study.

Montazari K. ${ }^{26}$ et al compared haemodynamic changes after use of facemask, LMA and tracheal tube. In their study compared with pre-induction and pre-insertion values, changes in HR and MAP values observed during 15 minutes after induction of general anaesthesia were statistically significant in all groups and the LMA group had a significantly lower HR and MAP than the other two groups (Repeated measures ANOVA: $\mathrm{P}<0.005)$, meanwhile the maximum mean changes in SBP, DBP and HR were more marked after ETT (SBP $15 \% \pm 11 \%$, DBP $10 \% \pm 13 \%$, HR $17 \% \pm 19 \%$ ) and FM (SBP $12 \% \pm 8 \%$, DBP $6 \% \pm 11 \%$, HR $13 \% \pm 7 \%$ ) than insertion of LMA (SBP-3\% $\pm 13 \%, \quad$ DBP $-5 \% \pm 16 \%, \quad H R \quad 4 \% \pm 13 \%) \quad(\mathrm{P}<0.005$, $\mathrm{P}<0.005$ and $\mathrm{P}<0.01$ for SBP and DBP and HR, respectively).

Our study also reveals less haemodynamic response by LMA insertion in comparison to ETT insertion. Also we have studied response after I-Gel insertion along with them, which shows even lesser response than LMA.

In a study conducted by Holden et al, ${ }^{16}$ they have compared intra-ocular pressure changes and cardiovascular changes using the laryngeal mask airway and tracheal tube. In their study, the mean change (SD) in IOP during airway placement compared to baseline was +1.8 (2.1) in LMA group and +6.8 (5.5) in tracheal tube group $(\mathrm{p}<0.0001)$. The mean change (SD) in HR during airway placement compared to baseline was +3.1 (10.2) in LMA group and +15.3 (14.3) in tracheal tube group $(\mathrm{p}<0.01)$. The mean change (SD) in SBP during airway placement compared to baseline was +2 (21.5) in LMA group and +16 (34.4) in tracheal tube group (P not significant). The mean change (SD) in DBP during airway placement compared to baseline was $+8.6(6)$ in LMA group and $+7.8(6)$ in tracheal tube group (P not significant).

In our study the IOP and HR increased with the tracheal group as in this study; however, our study also showed significant increase in SBP and DBP which is inconsistent with this study. The BP readings were not instantaneous and although there was not a significant difference between the two groups with regard to the BP, the trend was for the systolic $\mathrm{BP}$ to be higher in the tracheal tube group. This lack of significance may have been due to the fact that the BP monitoring was not continuous. We thus conclude that in a detailed study of 120 patients, insertion of I-Gel showed the least rise in IOP and haemodynamic parameters as compared to the C-LMA and ETT insertion.

\section{CONCLUSION}

Laryngoscopic endotracheal intubation produces statistically significant rise in intraocular pressure and haemodynamic parameters as compared to supraglottic airway devices in normotensive individuals scheduled for various elective surgeries.

Among the supraglottic airway devices, the classical laryngeal mask airway also produces statistically significant rise in intraocular pressure and haemodynamic parameters; however, this increase is significantly less than laryngoscopic endotracheal intubation.

I-GEL produces no significant increase in the intraocular pressure as well as haemodynamic parameters. As LMA produces minimal and I-GEL the least increase in intraocular pressure and haemodynamic response compared to tracheal intubation, these supraglottic devices can be used in selected elective surgical cases where these stress responses may be undesirable and better avoided.

\section{REFERENCES}

1. Forbes AM, Dally FG. Acute hypertension during induction of anaesthesia and endotracheal intubation in man. $\mathrm{Br} \mathrm{J}$ Anaesth 1970;42(7):618-24.

2. Mehernoor F Watcha, Paul F White, Lawrence Tychsen, et al. Comparative effects of laryngeal mask airway and endotracheal tube insertion on intraocular pressure in children. Anaesth Analg 1992;75(3):355-60.

3. Wynands JE, Crowell DE. Intraocular tension in association with succinylcholine and endotracheal intubation: a preliminary report. Can Anaes Soc J 1960;7(1):39-43.

4. Syed Altaf Bukhari, Imtiaz Naqash, Javed Zargar, et al. Pressor responses and intraocular pressure changes following insertion of laryngeal mask airway: comparison with tracheal tube insertion. Indian $\mathrm{J}$ of Anaesth 2003;47(6):473-5.

5. Richez B, Saltelf L, Banchereaur, et al. A new single use supraglottic airway device with a noninflatable cuff and an esophageal vent: an observational study of the I-Gel. Anaesth Analg 2008;106(4):1137-9.

6. Pennant JH, White PF. The laryngeal mask airway: its uses in anaesthesiology. Anaesthesiology 1993;79(1):144-63.

7. Kannaujia A, Srivastava U, Saraswat N, et al. A preliminary study of I-Gel: a new supraglottic airway. Indian Journal of Anaesthesia 2009;53(1):52-6.

8. Ghai B, Sharma A, Akhtar S. Comparative evaluation of intraocular pressure changes subsequent to insertion of laryngeal mask airway and endotracheal tube. Journal of postgraduate medicine 2001;47(3):181-4.

9. Robinson R, White M, McCann P, et al. Effect of anaesthesia on intraocular blood flow. British Journal of Ophthalmology 1991;75(2):92-3.

10. Duncalf D, Foldes F. Effect of anaesthetic drugs and muscle relaxants on intraocular pressure. In: Smith RB, ed. Anaesthesia in ophthalmology. Int Ophthalmol Clin 1973;13(2):21-34.

11. Kaskel D, Baumgart W, Metzler U, et al. Blood pressure, blood flow and intraocular pressure. Ophthalmic Res 1974;6(5-6):338-45.

12. Drenger B, Pe'er J, Ben Ezra D, et al. The effect of intravenous lidocaine on the increase in intraocular pressure induced by tracheal intubation. Anaesth Analg 1985;64(12):1211-3.

13. Dermot F Murphy. Anaesthesia and intraocular pressure. Anaesth Analgesia 1985;64(5):520-30.

14. Drenger B, Pe'er J. Attenuation of ocular and systemic responses to tracheal intubation by intravenous lignocaine. British Journal of Ophthalmology 1987;71(7):546-8. 
15. Mostafa S, Wiles J, Dowd T, et al. Effects of nebulized lignocaine on the intraocular pressure responses to tracheal intubation. Br J Anaesth 1990;64(4):515-7.

16. Holden R, Morsman $\mathrm{CD}$, Butler J, et al. Intra-ocular pressure changes using the laryngeal mask airway and tracheal tube. Anaesthesia 1991;46(11):922-4.

17. Lamb K, James MFM, Janicki P. The laryngeal mask airway for intraocular surgery: effects on for intraocular pressure and stress responses. Br J Anaesth 1992;69(2):143-7.

18. Bagshaw ONT, Southee R, Ruiz K. A comparison of the nasal mask and the nasopharyngeal airway in paediatric chair dental anaesthesia. Anaesthesia 1997;52(8):78696.

19. Duman A, Ogun CO, Okesli S. The effect on intraocular pressure of tracheal intubation or laryngeal mask airway insertion during sevoflurane anaesthesia in children without the use of muscle relaxants. Paed Anaesth 2001;11(4):421-4.

20. Levitan R, Kinkle W. Initial anatomic investigations of the I-Gel airway: a novel supraglottic airway without inflatable cuff. Anaesthesia 2005;60(10):1022-6.

21. Helmy AM, Atef HM, El-Taher EM, et al. Comparative study between I-Gel, a new supraglottic airway device, and classical laryngeal mask airway in anaesthetized spontaneously ventilated patients. Saudi J Anaesth 2010;4(3):131-6.
22. Ismail SA, Bisher NA, Kandil HW, et al. Intraocular pressure and haemodynamic responses to insertion of the I-Gel, laryngeal mask airway or endotracheal tube. Eur J Anaesthesiol 2011;28(6):443-8.

23. Agrawal G, Agarwal M, Taneja S. A randomized comparative study of intraocular pressure and haemodynamic changes on insertion of proseal laryngeal mask airway and conventional tracheal intubation in paediatric patients. Journal of Anaesthesiology Clinical Pharmacology 2012;28(3):326-9.

24. Maharjan SK. The haemodynamic and ventilatory responses with I-Gel, laryngeal mask airway and tracheal intubation during laparoscopic cholecystectomy. Journal of Kathmandu Medical College 2012;1(2):84-90.

25. Jindal P, Rizvi A, Sharma JP. Is I-Gel a new revolution among supraglottic airway devices? a comparative evaluation. MEJ Anaesth 2009;20(1):53-8.

26. Montazari K, Naghibi K, Hashemi SJ. Comparison of haemodynamic changes after insertion of LMA, facemask and endotracheal intubation. Acta Medica Iranica 2004;42(6):432-40. 